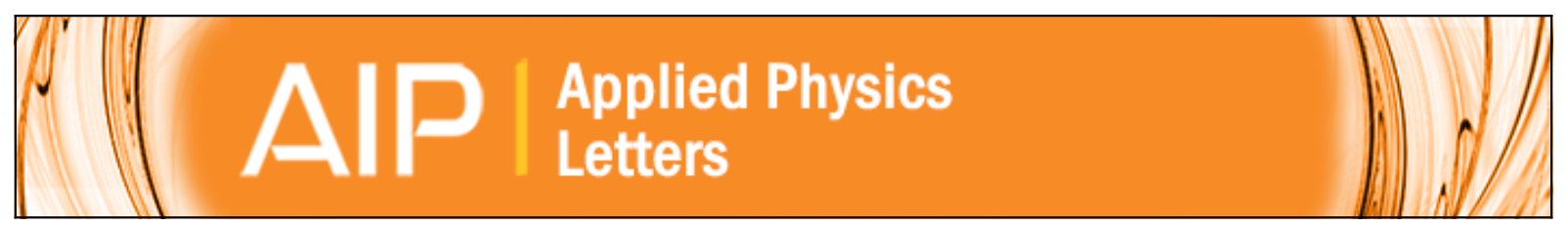

\title{
Direct synthesis of highly textured Ge on flexible polyimide films by metal-induced crystallization
}

N. Oya, K. Toko, N. Saitoh, N. Yoshizawa, and T. Suemasu

Citation: Applied Physics Letters 104, 262107 (2014); doi: 10.1063/1.4887236

View online: http://dx.doi.org/10.1063/1.4887236

View Table of Contents: http://scitation.aip.org/content/aip/journal/apl/104/26?ver=pdfcov

Published by the AIP Publishing

\section{Articles you may be interested in}

Low-temperature $\left(180^{\circ} \mathrm{C}\right)$ formation of large-grained $\mathrm{Ge}(111)$ thin film on insulator using accelerated metalinduced crystallization

Appl. Phys. Lett. 104, 022106 (2014); 10.1063/1.4861890

Improving the quality factor of polycrystalline Si thin-film micromechanical resonators by metal-induced lateral crystallization using biomineralized Ni nanoparticles

Appl. Phys. Lett. 103, 223103 (2013); 10.1063/1.4833875

Nucleation and growth kinetics during metal-induced layer exchange crystallization of Ge thin films at low temperatures

J. Appl. Phys. 111, 044908 (2012); 10.1063/1.3682110

Modified metal-induced lateral crystallization using amorphous Ge Si layered structure

Appl. Phys. Lett. 85, 899 (2004); 10.1063/1.1780595

In situ observation of nickel metal-induced lateral crystallization of amorphous silicon thin films

Appl. Phys. Lett. 80, 944 (2002); 10.1063/1.1447014

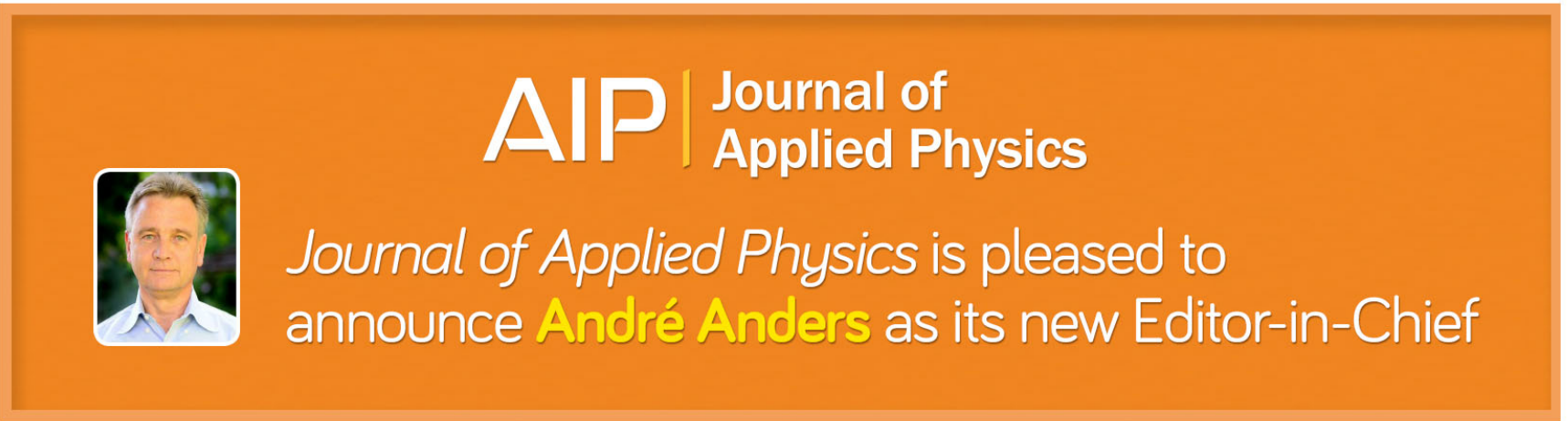




\title{
Direct synthesis of highly textured Ge on flexible polyimide films by metal-induced crystallization
}

\author{
N. Oya, ${ }^{1}$ K. Toko, ${ }^{1, a)}$ N. Saitoh, ${ }^{2}$ N. Yoshizawa, ${ }^{2}$ and T. Suemasu ${ }^{1}$ \\ ${ }^{1}$ Institute of Applied Physics, University of Tsukuba, 1-1-1 Tennodai, Tsukuba, Ibaraki 305-8573, Japan \\ ${ }^{2}$ Electron Microscope Facility, TIA, AIST, 16-1 Onogawa, Tsukuba 305-8569, Japan
}

(Received 3 June 2014; accepted 24 June 2014; published online 3 July 2014)

\begin{abstract}
The highly (111)-textured Ge thin film (50-nm thickness) is demonstrated on a flexible polyimide film via the low-temperature crystallization $\left(325^{\circ} \mathrm{C}\right)$ of amorphous $\mathrm{Ge}$ using $\mathrm{Al}$ as a catalyst. Covering the polyimide with insulators significantly improved the crystal quality of the resulting Ge layer. In particular, SiN covering led to $97 \%$ (111)-oriented Ge with grains $200 \mu \mathrm{m}$ in size, two orders larger than the grain size of polycrystalline Ge directly formed on the polyimide film. This achievement will give a way to realize advanced electronic and optical devices simultaneously allowing for high performance, inexpensiveness, and flexibility. (C) 2014 AIP Publishing LLC.

[http://dx.doi.org/10.1063/1.4887236]
\end{abstract}

Flexible electronics based on plastic substrates have attracted attention in recent years because of their light weight, handleability, and inexpensiveness. ${ }^{1}$ Using organic materials, easily formed below the softening temperature of plastic substrates (e.g., polyimide: $400^{\circ} \mathrm{C}$ ) has been the mainstream of flexible-device technology. ${ }^{2}$ However, the device performance of organic materials is still inferior as compared with that of inorganic materials. In line with this, the study of $\mathrm{Ge}$ on plastic is recently accelerated because of its attractive electrical and optical properties. ${ }^{3-5}$ The (111)textured $\mathrm{Ge}$ is specifically desirable, because it provides a high carrier mobility for metal-oxide-semiconductor transistors $^{6,7}$ and acts as an epitaxial template for group III-V compound semiconductors, aligned nanowires, and spintronics materials. $^{8-10}$

Transferring single-crystal Ge membranes to plastic substrates has been widely investigated for fabricating highperformance flexible thin-film transistors, photodetectors, and solar cells. ${ }^{3-5}$ The transfer techniques, however, have difficulties in lowering the process cost and fabricating large-area devices such as displays and solar cells. This motivates researchers to directly synthesize high-quality Ge on plastic below the softening temperatures of plastics. Because the solid-phase crystallization of amorphous $\mathrm{Ge}(\mathrm{a}-\mathrm{Ge})$ requires temperatures higher than $400{ }^{\circ} \mathrm{C},{ }^{11,12}$ metal-induced crystallization has gained much attention as a way to lower the crystallization temperature of a-Ge. ${ }^{13-21}$ Some researchers achieved polycrystalline Ge (poly-Ge) on plastic substrates; however, they had problems on the crystal quality of the resulting Ge layer or the expensive catalytic metals. ${ }^{19-21}$

Recently, we found that using $\mathrm{Al}$ as a catalyst and controlling annealing temperature $\left(180-375^{\circ} \mathrm{C}\right)$ allowed for large-grained, (111)-oriented poly-Ge on glass via exchanges between the Ge and Al layers. ${ }^{22,23}$ Such layer exchange is a well-known technique for producing polycrystalline $\mathrm{Si}$ on glass. ${ }^{24-28}$ Moreover, the crystal quality of the Ge layer was improved by selecting the substrate materials where Ge nucleates heterogeneously. ${ }^{29,30}$ By utilizing these knowledge,

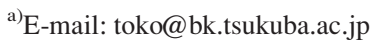

we investigate the Al-induced crystallization (AIC) of a-Ge and demonstrate the (111)-textured $\mathrm{Ge}$ of high-quality directly formed on a flexible plastic film.

Figure 1 schematically shows the process of sample preparation. We employed polyimide films as substrates because polyimides have a relatively high heat-resistance $\left(\sim 400^{\circ} \mathrm{C}\right.$ ) among flexible plastics. ${ }^{4}$ The thickness of the polyimide films were relatively large $(125 \mu \mathrm{m})$, which is important for laboratorial AIC as described later. The polyimide substrates were covered by nothing, $\mathrm{SiO}_{2}$, or $\mathrm{SiN}$ (100-nm thickness each); and, respectively, named samples A-C. 50-nm-thick Al layers were prepared on those samples, and then exposed to air for $10 \mathrm{~min}$ to form native $\mathrm{AlO}_{\mathrm{x}}$ membranes as diffusion limiting layers, ${ }^{24,25,27}$ followed by preparing 45-nm-thick a-Ge layers. All depositions were carried out at room temperature using a RF magnetron sputtering with Ar plasma. The samples were then annealed at $325^{\circ} \mathrm{C}$ in $\mathrm{N}_{2}$ for $100 \mathrm{~h}$ to induce layer exchange between $\mathrm{Ge}$ and $\mathrm{Al}$. For comparison, AIC-Ge on a $\mathrm{SiO}_{2}$ substrate was also prepared under the same conditions as samples A-C except the substrate, named sample $\mathrm{D}$. By removing the $\mathrm{Al}$ and $\mathrm{AlO}_{\mathrm{x}}$ layers using an $\mathrm{HF}$ solution (HF: $1.5 \%$ ) for $1 \mathrm{~min}$, bared
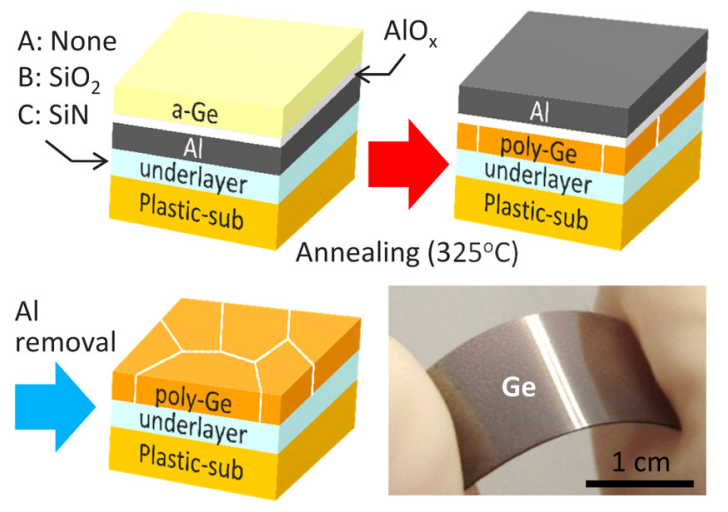

FIG. 1. Schematic of the preparation procedure of samples A-C, with polyimide substrates covered with nothing, $\mathrm{SiO}_{2}$, and $\mathrm{SiN}$, respectively. The photograph shows the resulting sample B. 
poly-Ge layers were obtained. The photograph of sample B is shown in Fig. 1, confirming the flexibility of the sample.

The crystal quality was evaluated by using a microprobe Raman scattering spectroscopy (spot size: $1 \mu \mathrm{m}$ and wavelength: $532 \mathrm{~nm}$ ) and a $\theta-2 \theta$ X-ray diffraction (XRD) measurement (spot size: $10 \mathrm{~mm}$ ). The crystal orientations and the grain sizes of the resulting poly-Ge layers were characterized by electron backscatter diffraction (EBSD) analysis. The detailed cross-sectional structure was investigated by the analytical transmission electron microscope (TEM), FEI Tecnai Osiris operated at $200 \mathrm{kV}$. Besides, the elemental composition of the sample was determined using an energy dispersive X-ray spectrometer (EDX). Cross-sectional TEM samples were prepared by conventional focused ion beam method.

Figure 2(a) shows the Raman spectra of samples A-C, where the background spectra originated from the polyimide substrates were subtracted. For all the samples, Raman peaks at around $290 \mathrm{~cm}^{-1}$ are clearly observed. These peaks indicate the crystallization of a-Ge, although they shift to approximately $10 \mathrm{~cm}^{-1}$ lower wavenumbers compared to the actual Ge optical phonon peak $\left(\sim 300 \mathrm{~cm}^{-1}\right) .^{9}$ This large wavenumber shift is a typical behavior in metal-induced crystallization including AIC, ${ }^{13,23}$ which is not completely understood but possibly relates to the residual catalytic metals in Ge. The $\theta-2 \theta$ XRD patterns in Fig. 2(b) show peaks at $27.2^{\circ}$ for samples B and C, corresponding to the (111) plane in relaxed Ge (JCPDS 04-0545). On the other hand, the XRD pattern of sample A shows no peak. In the $\theta-2 \theta$ XRD measurement of Ge thin films $(<50 \mathrm{~nm})$, peaks can be hardly observed as long as the films do not have preferential crystal orientation. Considering both results of the XRD and Raman measurements, we can conclude that sample A has a randomly oriented crystalline Ge layer, while samples B and C
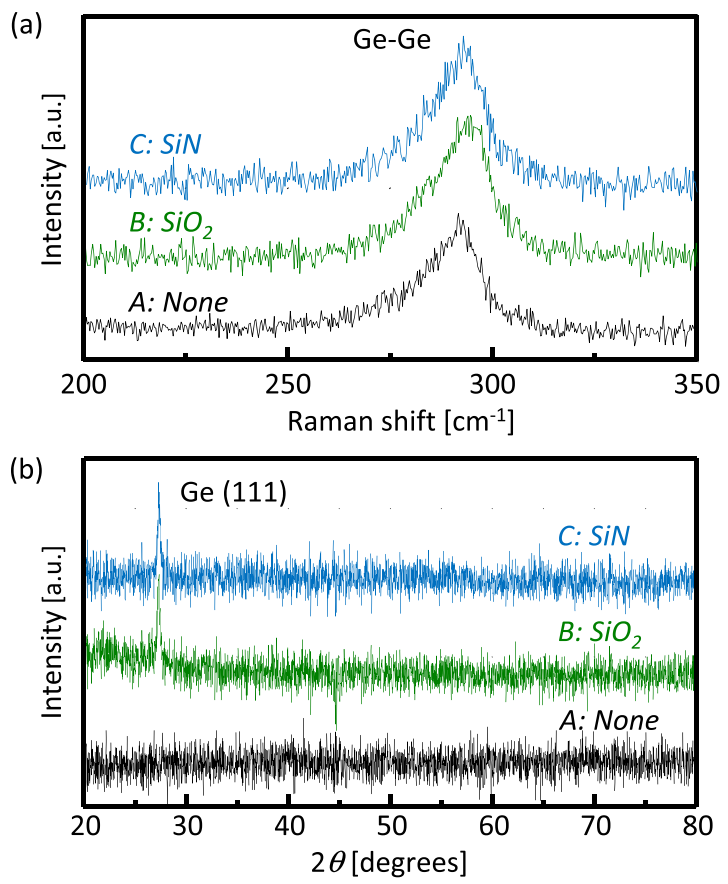

FIG. 2. (a) Raman scattering spectra and (b) $\theta-2 \theta$ XRD patterns of the resulting samples A-C. In the Raman spectra, the background spectra originated from the polyimide substrates were subtracted. have preferentially (111)-oriented Ge. Thus, the a-Ge layers were crystallized for all samples at as low as $325^{\circ} \mathrm{C}$ owing to the catalytic Al.

We investigated the detailed crystal orientation and grain size of $\mathrm{Ge}$ for samples $\mathrm{A}-\mathrm{C}$ using EBSD analysis. Figures 3(a)-3(f) show the EBSD images in the normal direction (ND) and the transverse direction (TD) to the sample surface. Figures 3(a) and 3(b) indicate that the Ge layer of sample A is randomly oriented and small-grained $(\sim 1 \mu \mathrm{m})$, consistent with the results of the Raman and XRD measurements shown in Fig. 2. In contrast, for samples B and $\mathrm{C}$, the Ge layers are highly (111)-textured as shown in Figs. 3(c) and 3(e). Such (111) orientation in AIC-Ge can be explained from the perspective of the appearance of the energetically stable plane. ${ }^{22,29-31}$ Moreover, as depicted in Figs. 3(d) and 3(f), their grain sizes are approximately two orders larger than that of sample A. Thus, covering the polyimide film with insulators can significantly improve the crystal quality of AIC-Ge.

Figure $3(\mathrm{~g})$ indicates that sample $\mathrm{B}$ has the comparable quality to sample $\mathrm{D}$, with a $\mathrm{SiO}_{2}$ substrate. Moreover, the grain size of sample $\mathrm{C}$ is twice as large as that of samples $\mathrm{B}$

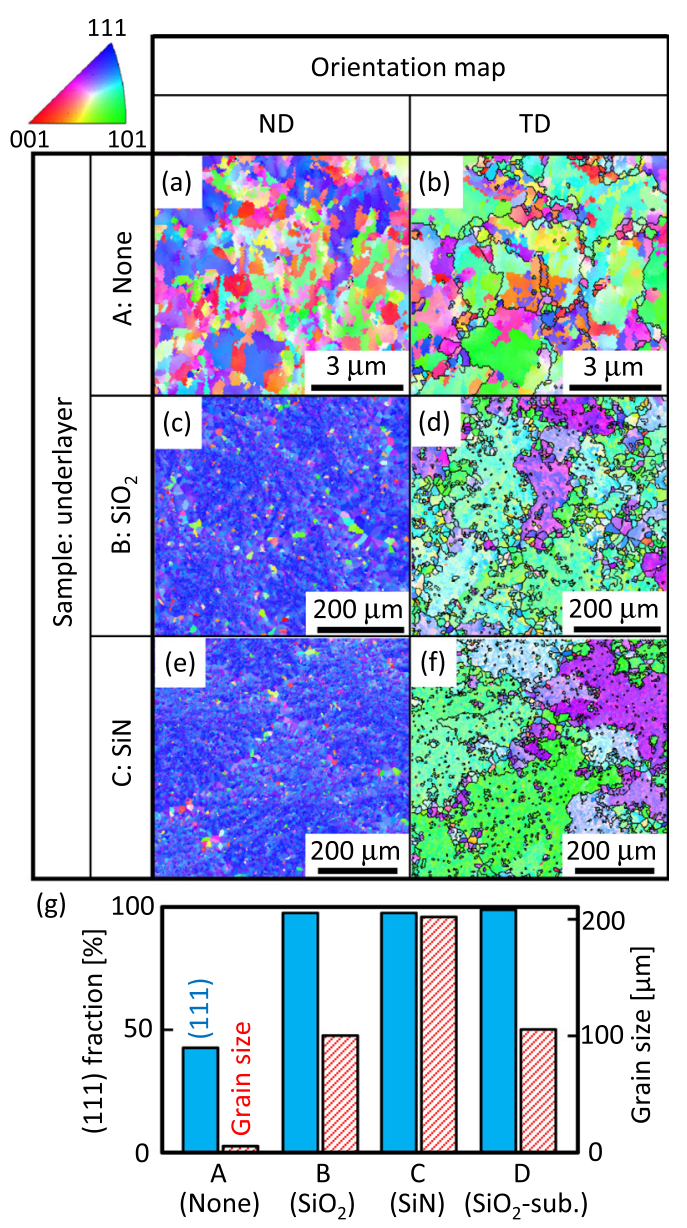

FIG. 3. EBSD images in ND and TD of the Ge layers for samples (a) and (b) - A, (c) and (d) - B , and (e) and (f) - C , where the ND and TD maps correspond to the same region. (g) (111) orientation fraction (left bar) and average grain size (right bar) of samples A-D calculated by EBSD analysis. By definition, the (111) fraction contains planes with tilts up to $15^{\circ}$ from the exact (111) plane. Coloration indicates crystal orientation, according to the legend. The black solid lines in the TD maps indicate random grain boundaries. 
and $\mathrm{D}$, indicating that $\mathrm{SiN}$ is more favorable than $\mathrm{SiO}_{2}$ for producing large-grained Ge layers. This result is consistent with the study on the liquid phase crystallization of Ge thin films. ${ }^{32}$ Thus, we achieved a $97 \%$ (111)-oriented Ge layer with grains approximately $200 \mu \mathrm{m}$ in size by covering the polyimide with $\mathrm{SiN}$. The mechanism of crystallization depending on the substrate, i.e., the effect of the underlying film, is discussed as follows. In metal-induced layer exchange, thin metal layers $(<100 \mathrm{~nm})$ allow Ge to heterogeneously nucleate at the substrates. ${ }^{29,30}$ Because the nucleation frequency and crystal orientation of Ge depend on the interfacial energy between $\mathrm{Ge}$ and its nucleation site, ${ }^{33}$ they should depend on the underlying material. The polyimide film employed in this work is considered to be incompatible with Ge probably owing to its composition or manufacturing process. That is why covering the polyimides with appropriate underlying layers, such as $\mathrm{SiO}_{2}$ or $\mathrm{SiN}$, effectively improved the crystal quality of the AIC-Ge. This means that the textured Ge layer can be obtained on any substrates as long as they are thermally stable under the AIC process $\left(180-350^{\circ} \mathrm{C}\right) .^{23}$

Now, a specific issue involved in flexible plastic substrates is mentioned. We found that the thickness of the polyimide substrate influences the crystal quality of AIC-Ge and attributed this behavior to the bending of the substrate. The polyimide films spontaneously bended during annealing, which was remarkable in the thin $(<100 \mu \mathrm{m})$ films. The bending likely strained the $\mathrm{Ge}$ and $\mathrm{Al}$ layers in AIC and then facilitated the Ge nucleation. This deteriorated the crystal quality of the resulting AIC-Ge layers. We therefore employed relatively thick $(125 \mu \mathrm{m})$ polyimide films in this work to avoid the bending effect. The industrial process including roll-to-roll process should be free from this issue because of the fixed substrates.

The detailed cross-sectional structure of sample $\mathrm{C}$ was investigated by using TEM. A bright-field TEM image in

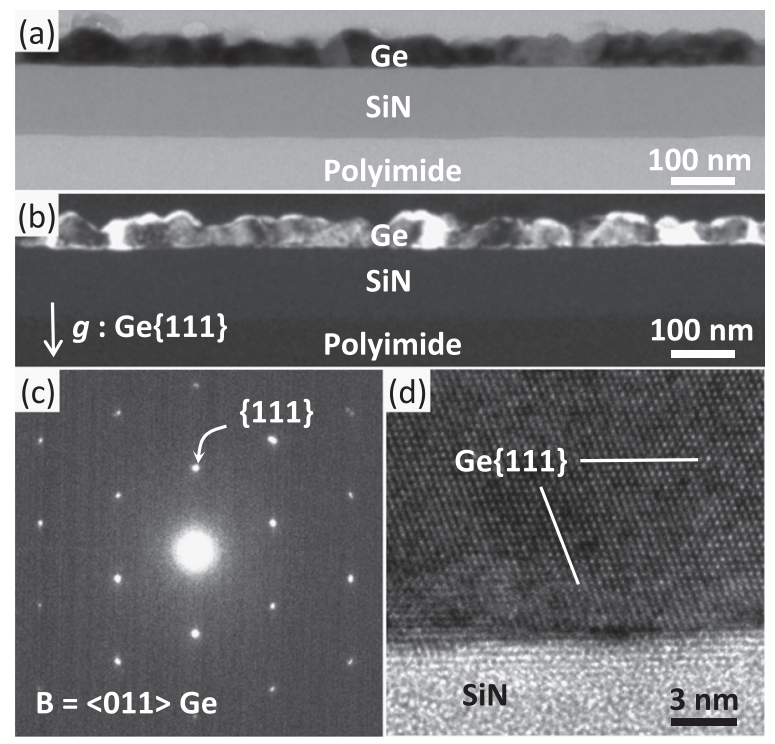

FIG. 4. Cross-sectional TEM images of the resulting sample C. (a) Brightfield image showing a uniform Ge layer stacked on SiN. (b) Dark-field image using the Ge $\{111\}$ plane reflection. (c) SAED pattern showing the Ge $\langle 011\rangle$ zone axis, taken from the bright-field image shown in (a). (d) High resolution image taken around the interface between $\mathrm{Ge}$ and $\mathrm{SiN}$.
Fig. 4(a) indicates a uniform Ge layer stacked on a SiN layer, which was confirmed by EDX analysis. Figure 4(b) shows a representative dark-field TEM image using the Ge $\{111\}$ plane reflection. The uniform bright contrast of the Ge layer indicates the single-crystal structure in this region including no obvious defect. The selected-area electron diffraction (SAED) pattern in Fig. 4(c) proves a single-crystal Ge which is (111) oriented in the normal direction relative to the substrate, consistent with the EBSD result shown in Fig. 3(e). Figure 4(d) shows a high-resolution TEM image of Ge layer in $\langle 011\rangle$ zone axis, indicating no-defect $\mathrm{Ge}$ with aligned $\{111\}$ planes. Moreover, the interface between the Ge and underlayer is uniform and contains no void structure owing to the simple crystallization process, demonstrating an advantage over conventional wafer bonding techniques.

In conclusion, we fabricated a high-quality crystalline Ge layer on a polyimide substrate via the transformation of Ge from amorphous to crystalline phase. Covering the substrate with SiN enabled the 97\% (111)-oriented Ge layer with grains as large as $200 \mu \mathrm{m}$ in size, exceeding the grain size of any other poly-Ge layers formed on insulating substrates. This achievement opens up the possibility for developing next-generation flexible devices simultaneously allowing for high performance and inexpensiveness.

This work was financially supported by the Japan Science Society and the Iwatani Naoji Foundation. The authors are grateful to Dr. N. Fukata of NIMS for assistance in Raman measurement. Some experiments were conducted at the International Center for Young Scientists in NIMS.

${ }^{1}$ J. A. Rogers, M. G. Lagally, and R. G. Nuzzo, Nature 477, 45 (2011).

${ }^{2}$ H. Sirringhaus, T. Kawase, R. H. Friend, T. Shimoda, M. Inbasekaran, W. Wu, and E. P. Woo, Science 290, 2123 (2000).

${ }^{3}$ G. Qin, T. Cai, H.-C. Yuan, J.-H. Seo, J. Ma, and Z. Ma, Appl. Phys. Lett. 104, 163501 (2014).

${ }^{4}$ W. S. Ho, Y.-H. Dai, Y. Deng, C.-H. Lin, Y.-Y. Chen, C.-H. Lee, and C. W. Liu, Appl. Phys. Lett. 94, 261107 (2009).

${ }^{5}$ D. Shahrjerdi, S. W. Bedell, C. Ebert, C. Bayram, B. Hekmatshoar, K. Fogel, P. Lauro, M. Gaynes, T. Gokmen, J. a. Ott, and D. K. Sadana, Appl. Phys. Lett. 100, 053901 (2012).

${ }^{6}$ T. Sasada, Y. Nakakita, M. Takenaka, and S. Takagi, J. Appl. Phys. 106, 073716 (2009).

${ }^{7}$ T. Nishimura, C. H. Lee, T. Tabata, S. K. Wang, K. Nagashio, K. Kita, and A. Toriumi, Appl. Phys. Express 4, 064201 (2011).

${ }^{8}$ E. P. a. M. Bakkers, J. a. van Dam, S. De Franceschi, L. P. Kouwenhoven, M. Kaiser, M. Verheijen, H. Wondergem, and P. van der Sluis, Nature Mater. 3, 769 (2004).

${ }^{9}$ N. Fukata, K. Sato, M. Mitome, Y. Bando, T. Sekiguchi, M. Kirkham, J.-I. Hong, Z. L. Wang, and R. L. Snyder, ACS Nano 4, 3807 (2010).

${ }^{10}$ K. Hamaya, H. Itoh, O. Nakatsuka, K. Ueda, K. Yamamoto, M. Itakura, T. Taniyama, T. Ono, and M. Miyao, Phys. Rev. Lett. 102, 137204 (2009).

${ }^{11}$ K. Toko, I. Nakao, T. Sadoh, T. Noguchi, and M. Miyao, Solid-State Electron. 53, 1159 (2009).

${ }^{12}$ C.-Y. Tsao, J. W. Weber, P. Campbell, G. Conibeer, D. Song, and M. A. Green, Sol. Energy Mater. Sol. Cells 94, 1501 (2010).

${ }^{13}$ I. Chambouleyron, F. Fajardo, and A. R. Zanatta, Appl. Phys. Lett. 79, 3233 (2001).

${ }^{14}$ B. Hekmatshoar, S. Mohajerzadeh, D. Shahrjerdi, and M. D. Robertson, Appl. Phys. Lett. 85, 1054 (2004).

${ }^{15}$ J. H. Park, P. Kapur, K. C. Saraswat, and H. Peng, Appl. Phys. Lett. 91, 143107 (2007).

${ }^{16}$ K. Toko, H. Kanno, A. Kenjo, T. Sadoh, T. Asano, and M. Miyao, Appl. Phys. Lett. 91, 042111 (2007).

${ }^{17}$ Z. M. Wang, J. Y. Wang, L. P. H. Jeurgens, F. Phillipp, and E. J. Mittemeijer, Acta Mater. 56, 5047 (2008). 
${ }^{18}$ M. Uenuma, B. Zheng, K. Bundo, M. Horita, Y. Ishikawa, H. Watanabe, I. Yamashita, and Y. Uraoka, J. Cryst. Growth 382, 31 (2013).

${ }^{19}$ S. Hu, A. F. Marshall, and P. C. McIntyre, Appl. Phys. Lett. 97, 082104 (2010).

${ }^{20}$ Z. Chen, Q. Li, D. Pan, H. Zhang, Z. Jiao, M. Wu, C.-H. Shek, C. M. L. Wu, and J. K. L. Lai, Mater. Today 14, 106 (2011).

${ }^{21}$ J.-H. Park, M. Miyao, and T. Sadoh, Jpn. J. Appl. Phys., Part 1 53, 020302 (2014).

${ }^{22}$ K. Toko, M. Kurosawa, N. Saitoh, N. Yoshizawa, N. Usami, M. Miyao, and T. Suemasu, Appl. Phys. Lett. 101, 072106 (2012).

${ }^{23}$ K. Toko, R. Numata, N. Oya, N. Fukata, N. Usami, and T. Suemasu, Appl. Phys. Lett. 104, 022106 (2014).

${ }^{24}$ O. Nast, T. Puzzer, L. M. Koschier, A. B. Sproul, and S. R. Wenham, Appl. Phys. Lett. 73, 3214 (1998).

${ }^{25}$ A. Sarikov, J. Schneider, J. Berghold, M. Muske, I. Sieber, S. Gall, and W. Fuhs, J. Appl. Phys. 107, 114318 (2010).
${ }^{26}$ B. I. Birajdar, T. Antesberger, B. Butz, M. Stutzmann, and E. Spiecker, Scr. Mater. 66, 550 (2012).

${ }^{27}$ M. Kurosawa, N. Kawabata, T. Sadoh, and M. Miyao, Appl. Phys. Lett. 95, 132103 (2009).

${ }^{28}$ D. Van Gestel, M. J. Romero, I. Gordon, L. Carnel, J. D’Haen, G. Beaucarne, M. Al-Jassim, and J. Poortmans, Appl. Phys. Lett. 90, 092103 (2007).

${ }^{29}$ K. Toko, K. Nakazawa, N. Saitoh, N. Yoshizawa, N. Usami, and T. Suemasu, CrystEngComm 16, 2578 (2014).

${ }^{30}$ J.-H. Park, T. Suzuki, M. Kurosawa, M. Miyao, and T. Sadoh, Appl. Phys. Lett. 103, 082102 (2013).

${ }^{31}$ A. A. Stekolnikov, J. Furthmüller, and F. Bechstedt, Phys. Rev. B 65, 115318 (2002).

${ }^{32}$ K. Toko, Y. Ohta, T. Tanaka, T. Sadoh, and M. Miyao, Appl. Phys. Lett. 99, 032103 (2011).

${ }^{33}$ P. Germain and K. Zellama, J. Appl. Phys. 50, 6986 (1979). 